\title{
Management of Papillary Carcinoma of Thyroid with Pulmonary Metastases in Institute of Nuclear Medicine and Allied Sciences, Rajshahi - Two Case Reports
}

Md. Kabiruzzaman Shah, Nasrin Begum, Mosharof Hossain, Parvez Ahmed, Sariful Islam Chawdhuary and M Shamim

Ahsan Institute of Nuclear Medcine and Allied sciences (INMAS), Rajshahi, Bangladesh

Correspondence Address : Md. Kabiruzzaman Shah, shahkabirz@ gmail.com, Director, Institute of Nuclear Medcine and Allied sciences (INMAS), Rajshahi, Bangladesh

\begin{abstract}
Papillary thyroid carcinoma with pulmonary metastasis is relatively uncommon which can be treated with radioactive iodine therapy. Here, our experiences with two cases of papillary thyroid carcinoma with pulmonary metastases in young patients are discussed.
\end{abstract}

\section{INTRODUCTION}

Thyroid cancers are the most common endocrine malignant tumors. Papillary thyroid carcinoma (PTC) is the most frequent type of differentiated thyroid carcinoma (DTC) with a ratio of $80 \%(1,2)$. PTC commonly metastasizes to regional lymph nodes. However, distant metastasis may occur in 5\% patients-lung and bones being the most common sites for distant metastases (3). Though the prognosis of the disease is excellent following radioiodine ablation/therapy, 10-year survival is approximately $25-40 \%$ in case of distant metastasis $(4,5)$.

Here we reported our experience with two cases of PTC having pulmonary metastasis.

\section{CASE REPORT -I}

A 23-year old unmarried female patient with papillary thyroid carcinoma (PTC) was referred to our institute in 2006. She underwent total thyroidectomy in July 2006. Post surgical evaluation showed high serum thyroid stimulating hormone (TSH) (60 mIU/L) and large dose whole body iodine scan showed small residual tissue in thyroid bed. She was treated with $138 \mathrm{mci}$ of radioactive iodine (I-131) in September 2006. She was kept in TSH suppression. Diagnostic whole body scan (DxWBS) was done one year after radioiodine ablation and was negative for metastasis. She was followed up regularly till 2009 and had no evidence of malignancy. Afterwards she was lost from follow up and returned to us in 2014 with fever, cough and occasional respiratory distress. She had history of irregular/inadequate intake of thyroxine for the last five years. On evaluation she was found hypothyroid with TSH $23.4 \mathrm{mIU} / \mathrm{L}$. Serum thyroglobulin $(\mathrm{Tg})$ was raised $(37.23 \mathrm{pg} / \mathrm{ml})$. Chest radiograph showed patchy opacities in both lung fields (Figure-1). DxWBS was done with $4 \mathrm{mCi}$ of I-131 which showed patchy radiotracer uptake in both lung fields suggesting iodine avid pulmonary metastases (Figure-2).

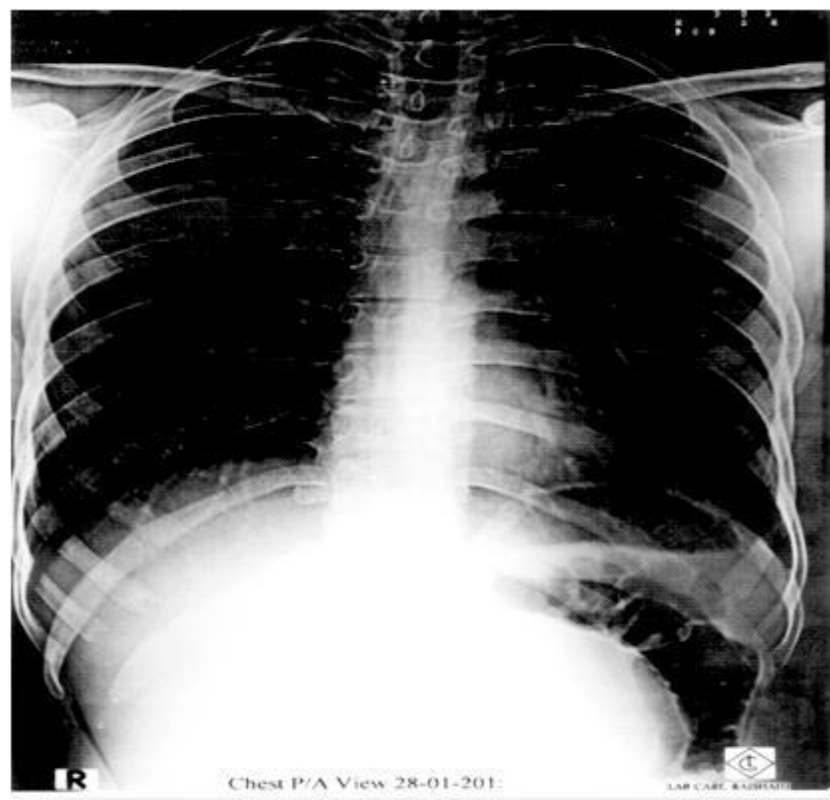

Figure 1: Chest X-ray showing ill defined patchy areas in both lungs 


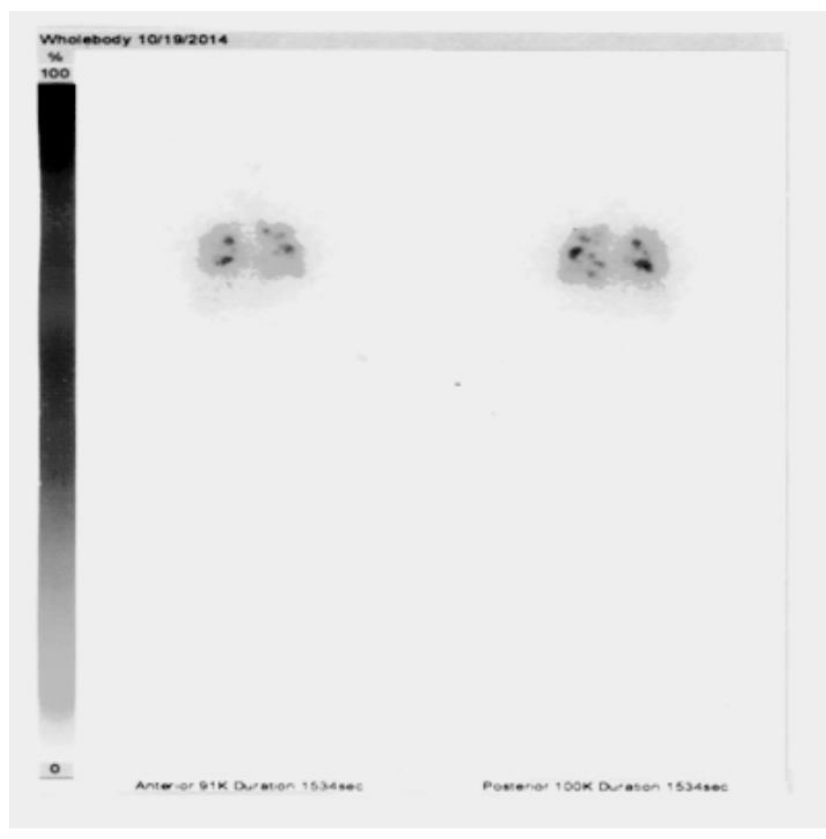

Figure 2: Whole body iodine scan showing bilateral lung uptake

Then she was given another dose of radioiodine therapy with 200 mci I-131 with steroid coverage. She was kept in optimum TSH suppression and strictly followed up. Gradually she became symptom free and serum Tg level returned to expected level. Chest X-ray was normal (Figure-4) and DxWBS done after one year in June 2015 was negative for persistent/recurrent disease (Figure-5).

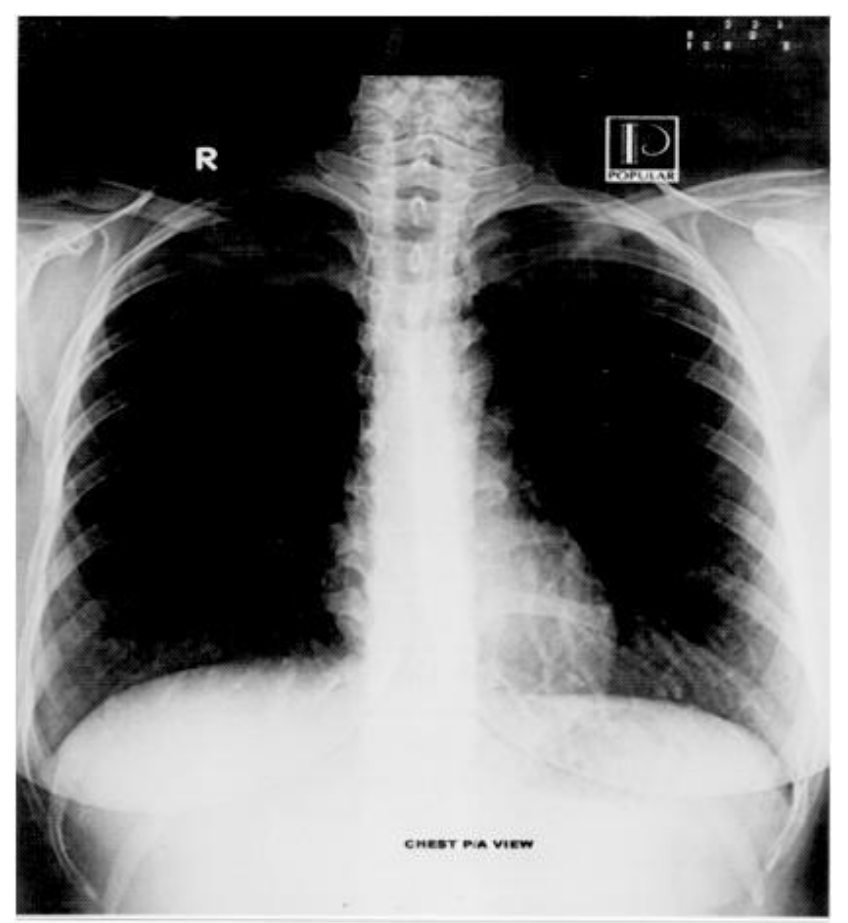

Figure 3: Normal chest X-ray after radioiodine therapy

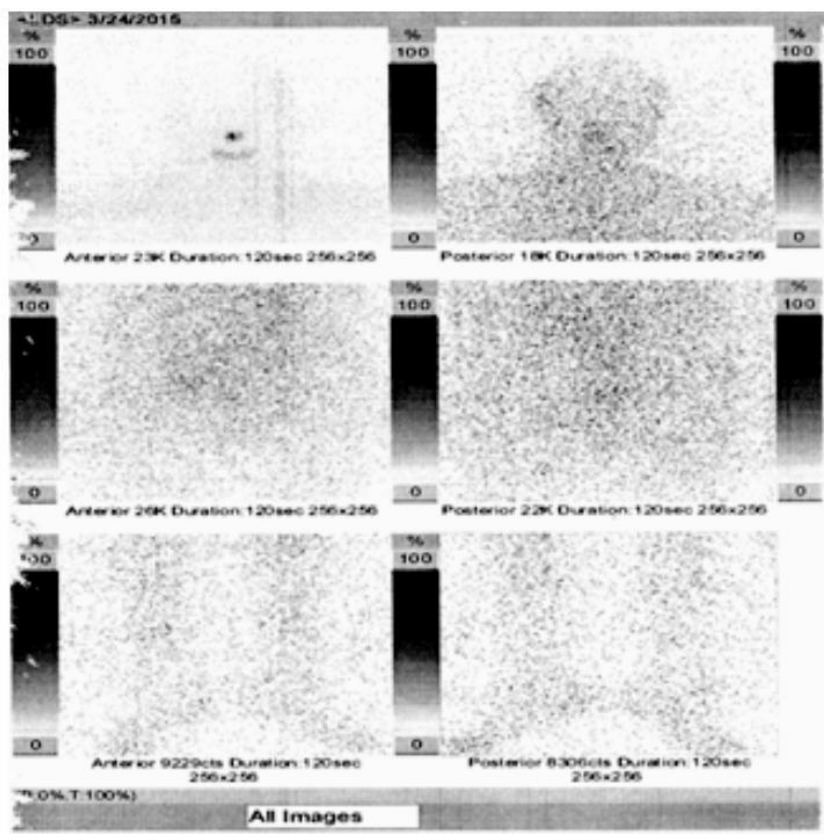

\section{Figure 4: Negative whole body scan}

after treatment CASE REPORT -2

A 20 Years old man with history of papillary carcinoma of thyroid and local nodal metastases was referred to our institute for radioactive iodine ablation. He underwent total thyroidectomy and radical neck dissection. He had some respiratory symptoms like cough, occasional respiratory distress and husky voice. His chest X-Ray revealed patchy opacity in upper zone of right lung. Post surgical evaluation showed high serum TSH (34.0 mIU/L) and high serum Tg level (42.81 ng/ml). DxWBS showed residual functioning thyroid tissue in thyroid bed and radioiodine uptake in upper part of right lung (Figure-5). He was treated with $200 \mathrm{mCi}$ of $\mathrm{I}-131$ with steroid coverage and thyroxine was started in suppressive dose from third post therapy day.

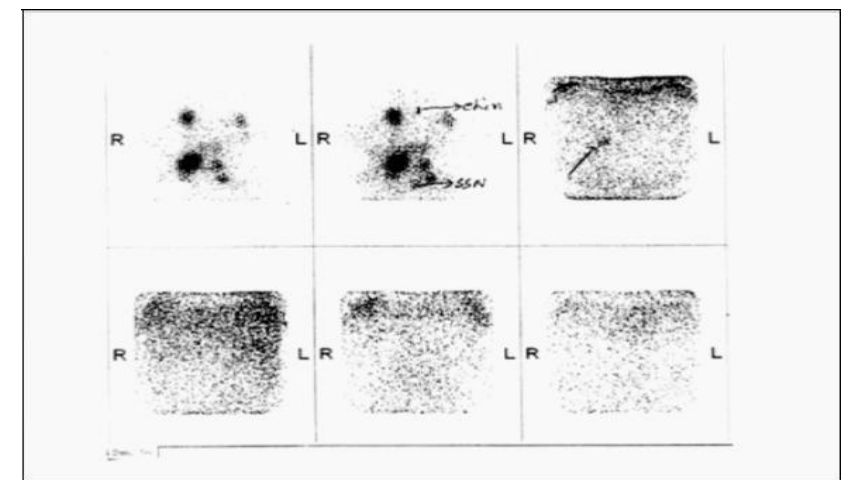

Figure 5: Whole body scan showing right lung uptake 


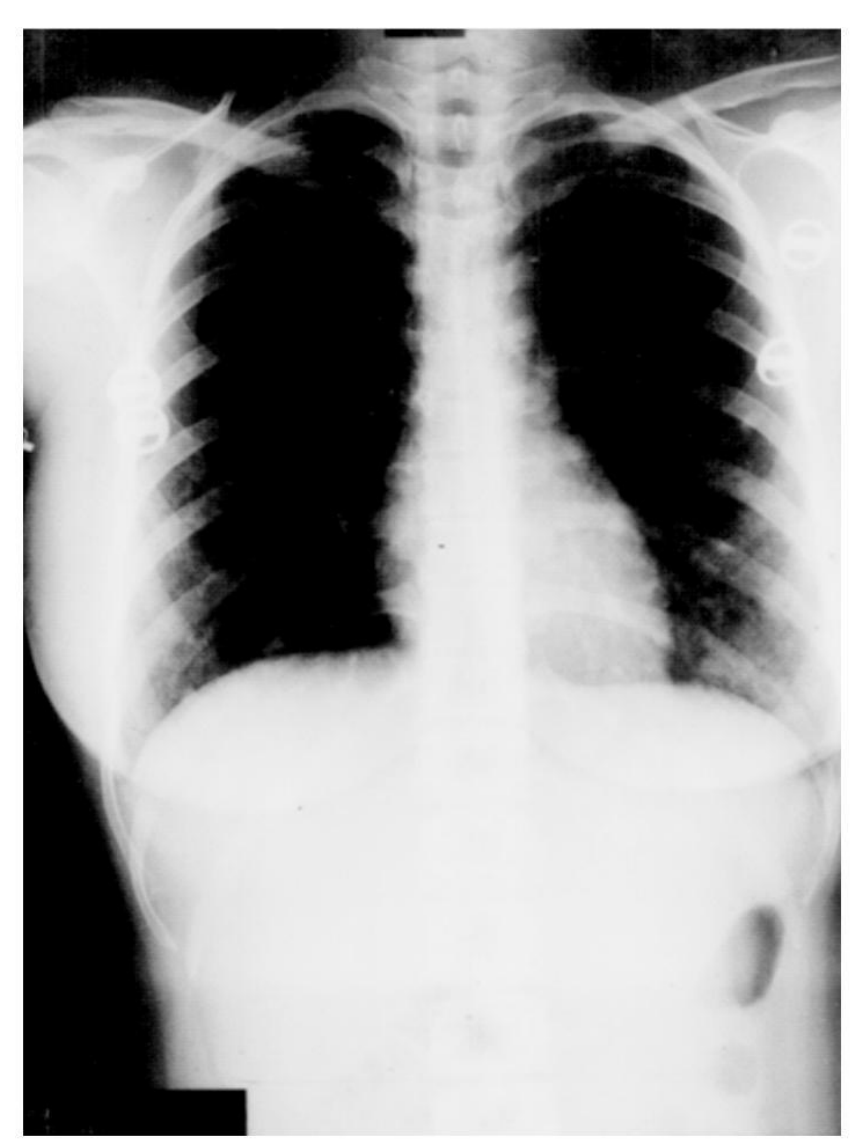

Figure 6: Normal Chest X-ray after treatment

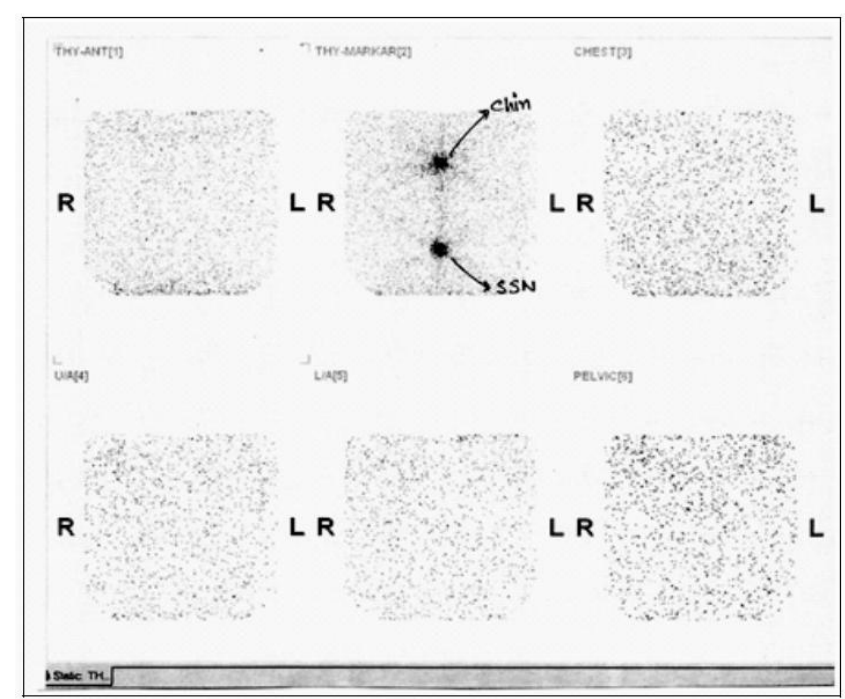

Figure 7: Negative whole body scan after treatment

His hospital stay was uneventful. First follow-up after 3 months, showed well suppressed TSH (0.19 $\mathrm{mIU} / \mathrm{L})$ and serum $\mathrm{Tg}$ returned to expected level (1.2 $\mathrm{ng} / \mathrm{ml}$ ). Chest X-Ray showed clear both lung fields. $\mathrm{He}$ was clinically normal. He was followed up regularly and DxWBS done after one year which revealed no abnormal uptake in thyroid bed as well as in lung field or any other part of the body.

\section{DISCUSSION}

Primary thyroid cancers are histologically divided in four groups: i) Well differentiated epithelial cancers, ii) Poorly differentiated epithelial thyroid cancer iii). Medullay thyroid cancer and iv) Rare thyroid tumors (lymphoma, sarcoma, squamous cell carcinoma etc). Of these differentiated thyroid carcinoma (DTC) papillary and follicular constitute the largest part with a rate of over $90 \%$ (6). Papillary thyroid cancer (PTC) is the most common type of differentiated thyroid cancers (3).

Treatment of differentiated thyroid carcinoma includes total/near total thyroidectomy followed by radioactive iodine ablation and TSH suppression by Thyroxine. In case of PTC the 10-year survival rate is $93 \%$ following total thyroidectomy and radioiodine ablation. It is one of the best treatable cancers. Young patients with small tumors without invasion have better prognosis (7). A thyroid carcinoma may be associated with a high TSH stimulation. A study conducted with rats reported the presence of long term elevated TSH levels resulting in a thyroid carcinoma with lung metastasis (8). TSH suppression is mandatory for good prognosis as it inhibits growth of cancer cells (9). First patient reported here, developed lung metastases after 5 years and during that period she was irregular in thyroxine intake and had high TSH level at the time of detection of lung metastases. About 10 to $15 \%$ of patients with papillary thyroid carcinoma develop distant metastases- $80 \%$ of which occur in lungs (10). Two distinct type of metastasis occur in lungs parenchyma - miliary metastasis and multiple nodules (11). Pulmonary metastases have good prognosis with I131 when they are undetectable in chest X-ray (12). One of our reported patients showed multiple nodules in both lung fields. The other had solitary pulmonary nodule and both of them had positive chest X-ray. 
Both of them showed good response to radioiodine ablation and became disease free after radioiodine therapy probably due to early detection and treatment.

\section{CONCLUSION}

Metastatic PTC is an uncommon condition that generally has a relatively good prognosis when diagnosed and treated easily. Adequate TSH suppression and regular follow up are mandatory for DTC patients following radioactive iodine ablation to prevent recurrence.

\section{REFERNCES}

1. Fagir JJ and Mitsides N. Molecular Pathology of Thyroid Cancer: Diagnostic and Clinical Implications. Best Practice and Research: Clinical Endocrinology and Metabolism 2008;22(6):955-59.

2. Y.E.R.E. Nikiforov. Thyroid Carcinoma: Molecular Pathways and Therapeutic Targets. Modern Pathology 2008;21(2):537-43.

3. Borschitz T, Eichhorn W, Fottner C, Sampson E. Diagnosis and Treatment of Pancreatic Metastases of a Papillary Thyroid Carcinoma. Thyroid 2010;20(1):93-98.

4. Dinneh ST, Valimaki MJ, Bergstralh EJ, Goellner JR, Gorman CA, Hav ID. Distant Metastases in Papillary Thyroid Carcinoma 100 Cases Observed at One Institution During 5 Decades. J Clin Endocrinol Metob 1995;80

(7):2041-50.
5. Casara D, Rubelloi D, Saladini G, Masarotto G, Favero A, Girelli ME, Busnardo B. Different Features of Pulmonary Metastases in Differentiated Thyroid Cancer: Natural History and Multivariate Statistical Analysis of Prognostic Variables. J Med 1993;34(10):1626-31.

6. Ringel MD and Ladenson PW. Controversies in the Follow up and Management of Well Differentiated Thyroid Cancer. Endocrine Related Cancer 2004;11(1):.97-116.

7. Kebebew E and Clark OH. Differentiated Thyroid Cancer: Complete Rational Approach. World Journal of Surgery 2000;.24(8):942-51.

8. Patel PC, Mallman B, Pellitteri PK, Woods EL. Papillary Thyroid Carcinoma Presenting with Massive Angioinvasion of Great Vessels of the Neck and Chest. Otolaryngol Head Neck Surgery 1997;117(S6):117-20.

9. Cooper DS, Doherty GM, Haugen BR, Kloos RT, Lee SL, Mandel SJ et al. Revised American Thyroid Association Management Guidelines for Patients with Thyroid Nodules and Differentiated Thyroid Cancer. Thyroid 2009;19(11):1167-1214.

10. Schlumberger MJ, Torlanto M. Papillary and Follicular Thyroid Carcinoma. Best Practice and Research Clinical Endocrinology Metabolism 2000;14(4):601-13.

11. Hoie J, steming AE, Kullmann G, Lindengaag M. Distant Metastases in Papillary Carcinoma. A Review of 91 Patients. Cancer 1998; 61(1):1-6.

12. Chen L, Shen Y, Luo Q, Yu Y, Lu H, Zhu R. Pulmonary Fibrosis Following Radioiodine Therapy of Pulmonary Metastases From Differentiated Thyroid Carcinoma. Thyroid 2010,20(3):337-41. 